\title{
Fracture Care Challenges in the Austere and Humanitarian Environments
}

\author{
Richard E. Gellman ${ }^{1}$
}

Published online: 26 April 2016

(C) Springer International Publishing AG 2016

\begin{abstract}
It is estimated that $80 \%$ of severe fractures occur in the developing world. Long bone fractures of the femur and tibia treated conservatively with traction and casts require prolonged hospital stays and still often leave patients with significant lifelong disability. Locked intramedullary nailing is the standard of care in the developed world for management of these fractures. Humanitarian fracture care in an austere environment often means limited or no access to implants, a reliable power supply and intraoperative radiography traditionally required for locked intramedullary nailing. Creative surgeons with extensive experience in humanitarian work are making significant contributions to improve the management of these complex fractures in the austere environment through education of local surgeons and innovations in orthopedic implants and instrumentation.
\end{abstract}

Keywords Locked intramedullary nailing $\cdot$ Femoral and tibial shaft fractures · Surgical Implantation Generation Network (SIGN)

\section{Introduction}

It is now well recognized that trauma is one of the fastest growing epidemics worldwide. Although the majority of the published trauma literature comes from the more developed countries with established trauma systems, trauma represents

This article is part of the Topical Collection on The Military Perspective

Richard E. Gellman

rgellman@me.com

1 Summit Orthopaedics, 501 N Graham St \#250, Portland, OR 97227, USA a significant burden in terms of death, disability, and cost in less developed or "third-world" areas. By 2030, road traffic injuries alone are predicted to become the third largest contributor to the global burden of disease [1]. The majority of these injuries occur in the developing world where road systems are poorly maintained and overcrowded and the number of people travelling in each vehicle or on each motorbike is significantly higher (Fig. 1).

Until recently, the World Health Organization has largely neglected the impact that trauma has on developing nations, prioritizing funds for infectious diseases such as malaria, tuberculosis, and HIV/AIDs [2]. Traditional thinking assumed injuries requiring surgical subspecialty care, and particularly costly supplies and equipment, were too expensive to treat in low- and middle-income (LMICs) countries.

Recent studies on the global burden of disease have shown that musculoskeletal injuries, as measured by disabilityadjusted life years (DALYs), are large compared to communicable diseases [3]. What this means is that surgically treating a femur fracture is as beneficial in terms of resource utilization as treating a malaria patient with medication and mosquito netting, even in resource-limited nations. In fact, the benefits in terms of overall quality of life, ability to work, and longevity likely are several orders of magnitude greater than treating many other non-traumatic diseases [4].

In the USA and other countries with more robust and advanced trauma systems, orthopedic care of fractures typically use modern management principles and state-of-the-art equipment and techniques. Most fractures of the femur or tibia that occur in the diaphyseal (shaft) portion are treated with early surgical correction. The standard treatment is a closed reduction or limited open reduction technique that relies on intraoperative radiography, power reaming, and stabilization with locked intramedullary nails. This has clearly been demonstrated to result in significantly faster recovery, earlier 


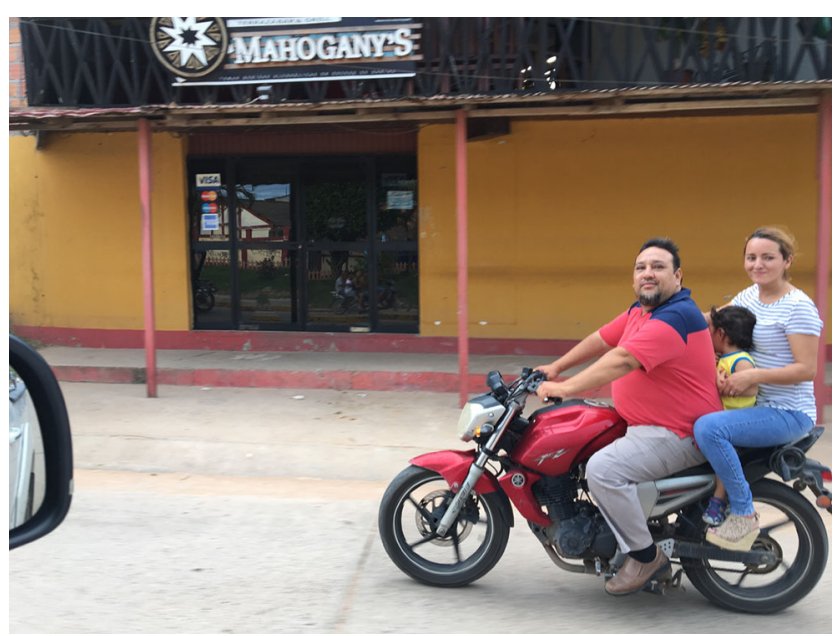

Fig. 1 Motorcycles or motorscooters are a common form of transportation in developing countries contributing to the rise in traumatic injuries

mobilization, and superior long-term functional outcomes. Unfortunately, throughout most developing nations, the management of femur and tibia shaft fractures remains limited to closed treatment with prolonged traction for femur fractures and long leg cast splinting and immobilization for tibia fractures [5] (Fig. 2).

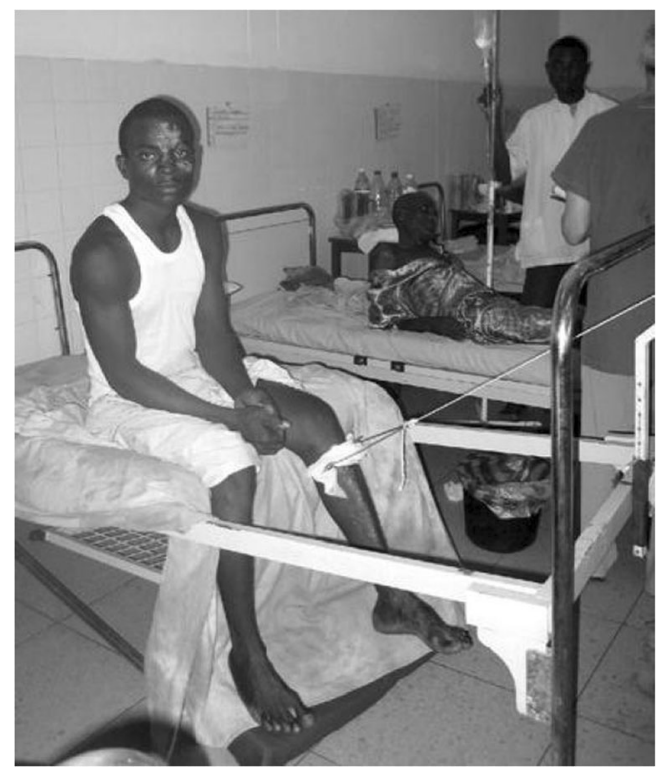

Fig. 2 This is an example of Perkin's traction for the treatment of femoral shaft fractures. Patients have a traction pin inserted into the proximal tibia with approximately $20 \mathrm{lbs}$ of weight applied. During the day, the end of the bed is removed and exercises in knee motion are begun immediately. Initially, there is a need of intensive physical therapy until patients are able to do the exercises on their own. At night, the lower end of the bed is replaced so patients are able to lie supine to sleep. Duration of traction, if all goes well, is approximately 8 weeks. If radiographs show fracture healing, patients are transitioned to partial weight bearing on crutches for another 4-8 weeks until satisfactory healing. Pin tract infections are common but can be treated with simple debridement. Malunion of the fracture is also a common sequelae
While closed treatment of femur and tibia fractures with traction is certainly safe in an austere environment, there are several reasons that make this an inferior treatment option. First, there is a prolonged hospital stay, on average 8 weeks for femoral shaft fractures until patients can be mobilized out of the hospital on crutches $[6,7]$. Traction wards were common in developed nations until the early 1980s when the development of locked intramedullary nailing replaced traction as the standard of care [8-10].

Secondly, locked intramedullary nailing of femur and tibia fractures has shown to improve healing or union rates and decrease rates of malunion or angular deformities and limb shortening [11-13]. In addition, prolonged or permanent knee joint stiffness is significantly decreased compared with simple splint or traction immobilization [14].

Similar excellent outcomes in terms of the safety and costeffectiveness of intramedullary nailing have been demonstrated in developing nations when surgeons have access to implants and the appropriate training $[15,16]$. However, this does require a significant up-front monetary investment in equipment and training, as well as continued recurring costs for the expensive hardware. These costs and equipment requirements, particularly for powered reaming devices and intraoperative fluoroscopy to provide radiologic guidance, represent an insurmountable barrier in many settings.

\section{The SIGN Organization}

In 1999, the Surgical Implantation Generation Network (SIGN) introduced a technology that allows orthopedic surgeons to treat femur and tibia fractures with locked intramedullary nailing without the need for image intensifiers, fracture tables, or power reaming [17•].

The SIGN organization was founded by Lew Zirkle, an orthopedic surgeon with extensive experience in humanitarian fracture care. After spending 25 years teaching and developing orthopedic training programs in Indonesia and Vietnam with CARE-medico and Orthopedics Overseas, Dr. Zirkle felt driven to develop a simplified and low-cost locked intramedullary nailing system that could be used in resourcepoor environments [18]. His goal was to create a system that could not only be safe and economically viable even in lowincome countries but that would offer outstanding short- and long-term outcomes comparable to the more expensive standard systems used in high-income settings.

The inspiration for the SIGN nail occurred in the mid 1990s when Dr. Zirkle met an Indonesion man who had been in traction for 3 years because his femur fracture had not healed, leaving him with a painful nonunion. The patient could not afford the implant necessary to stabilize and heal his fracture. Dr. Zirkle's work in Indonesia was a model for program development in orthopedic education, with well-trained 
surgeons available as a result of his efforts there. Despite this fact, well-trained orthopedists in developing nations still needed reliable tools and implants [18].

Dr. Zirkle worked for several years trying different methods to insert interlocking screws without the use of radiography but was unsuccessful. Then, in 1999, he collaborated with Randy Huebner, a talented mechanical engineer and founder of Acumed, an orthopedic implant company based in Portland, Oregon. Together, they developed the first set of SIGN instruments and nails that Dr. Zirkle first used to treat fractures in Vietnam in the late 1999.

The unique feature of the SIGN nail instrumentation is a rigid external targeting arm that attaches to the nail and allows placement of the distal interlocking screws without radiography (Fig. 3). Oval-shaped interlocking holes in the SIGN nails are localized by a precise, three-step series of a hand drill and two "slot finders" to identify the oval holes. Rotation of the slot finder, termed the "SIGN feel," verifies its position within the nail and allows completion of drilling through the far cortex of the bone (Fig. 4).

The SIGN nail is constructed of solid stainless steel to reduce cost and increase strength. This feature prevents closed nailing over a guidewire; however, this technique does not apply to resource-poor hospitals where the nail is used. Biomechanical comparisons with cannulated or hollow nails confirmed that a 9-mm solid SIGN nail has superior strength compared to $10 \mathrm{~mm}$ hollow nails [19]. While the SIGN nail is a low-cost implant that can be distributed to resource-poor nations, it is Food and Drug Administration (FDA) approved for use in the USA, confirming that it meets the highest standards for quality, materials, and manufacturing [17॰].

In addition to learning the technique to place the interlocking screws, a surgeon who wishes to perform open nailing without power reamers or intraoperative radiography will be challenged to develop new and useful skills. The first is a thorough understanding of the anatomy at the proximal femur and tibia since the start points for introducing hand reamers comes from bony landmarks not seen under an image intensifier. Second, the surgeon will develop the feel (and strength) needed to pass hand reamers down the intramedullary canals of the femur and tibia, which should

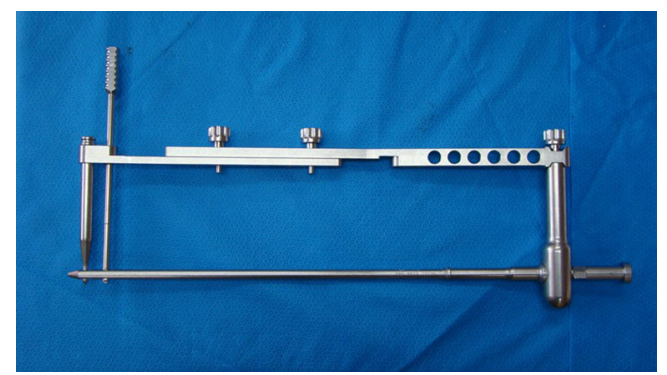

Fig. 3 The SIGN external targeting guide. The surgeon inserts cannulas and drills that allow placement of the interlocking screws at the end of the nail

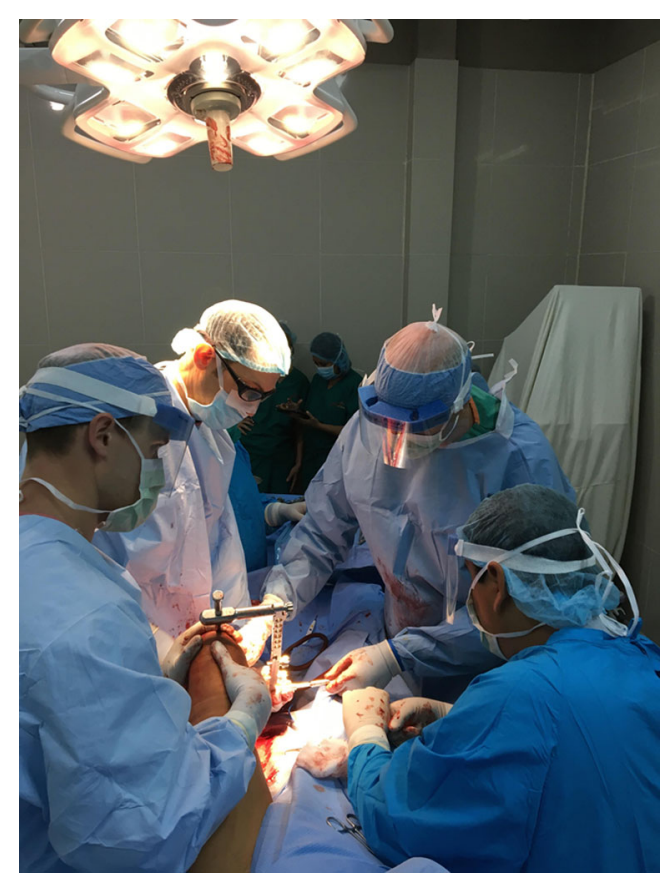

Fig. 4 The SIGN "feel." The surgeon on the right has the slot finder cannula inserted into the medial aspect of the tibia. By rotating the cannula $20^{\circ}$ he is able to feel the cannula within the oval hole of the nail

be approximately $2 \mathrm{~mm}$ larger than the nail outer diameter (Fig. 5).

The SIGN technique requires making an incision at the fracture site to feel the fracture ends or to perform an open reduction of the fracture in most cases (Figs. 6, 7, 8, and 9, case example). Over the last 10 years, trauma fellowshiptrained orthopedic surgeons in the USA have moved toward

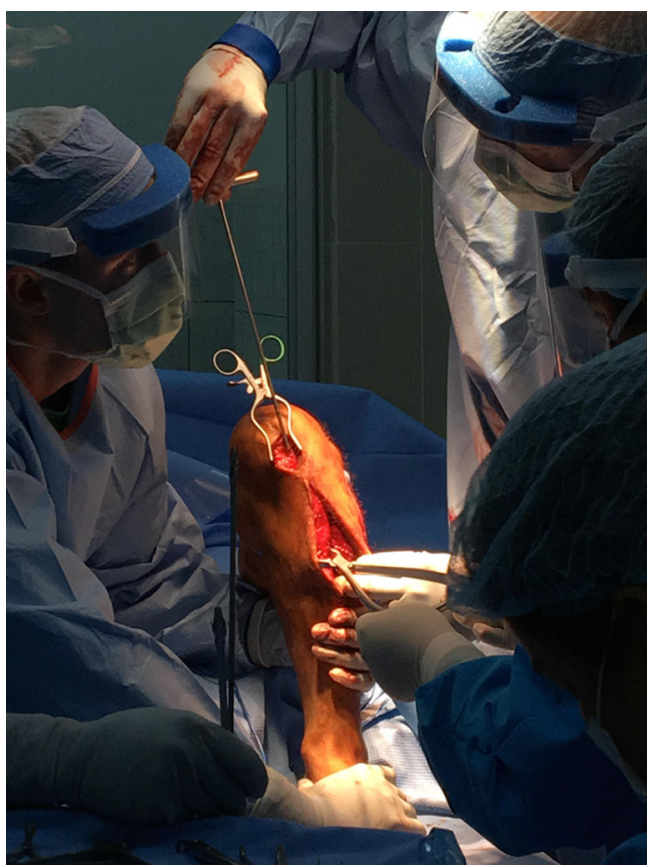

Fig. 5 Open reduction and hand reaming shown below are two key features of the SIGN technique 


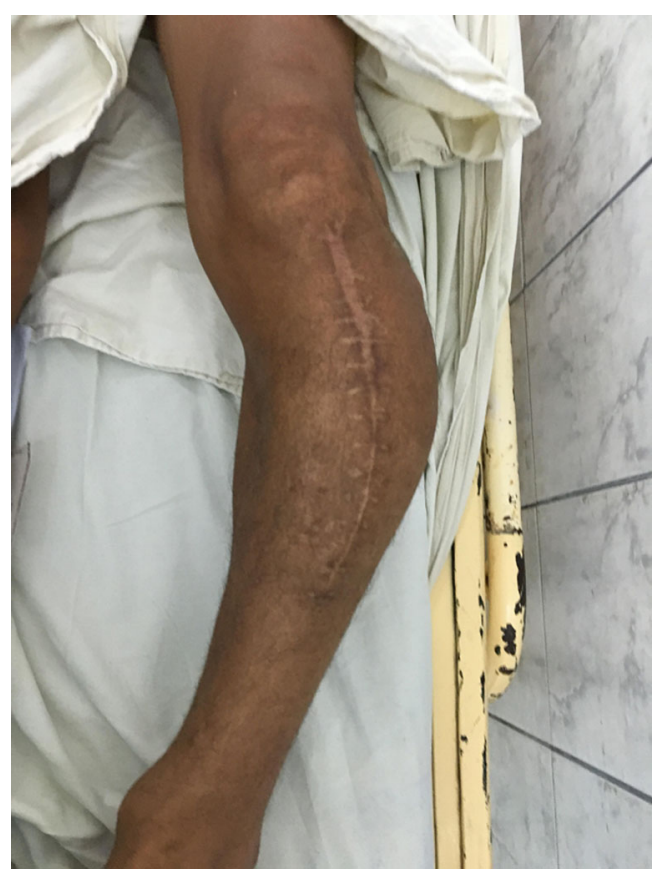

Fig. 6 The left leg of a 51-year-old Peruvian man with a nonunion and malunion of his tibia

performing limited open reductions in closed tibia fractures to improve fracture alignment prior to placement of intramedullary nails without an increase in infection or nonunion rates [20]. Limited open reductions in the femur are quite safe from an infection standpoint but may add to greater blood loss compared to closed reductions with intraoperative radiography. The clinical decision to proceed with open nailing will be influenced by the patient's starting blood

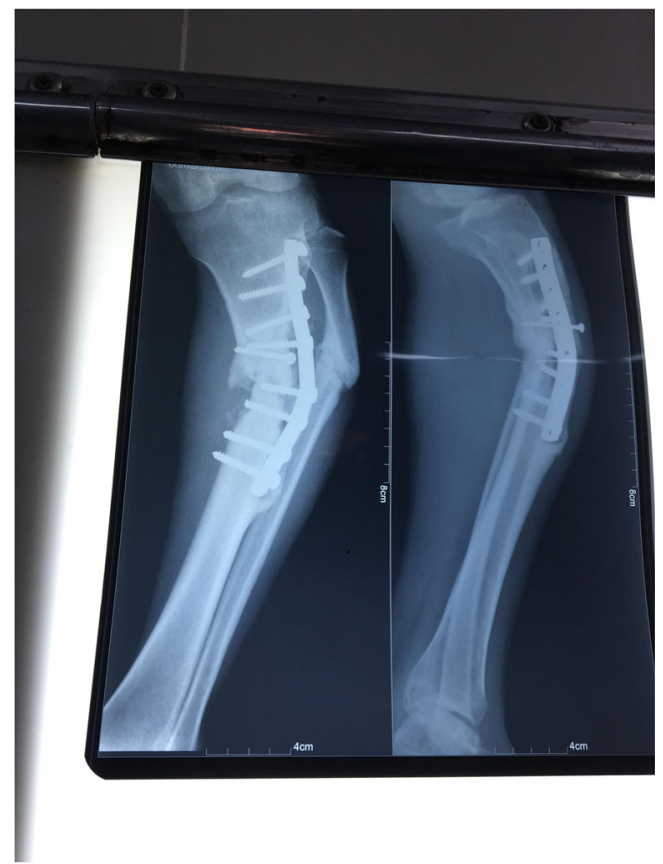

Fig. 7 Pre operative radiograph of the varus deformity and broken plate removed and correction of deformity with a SIGN nail

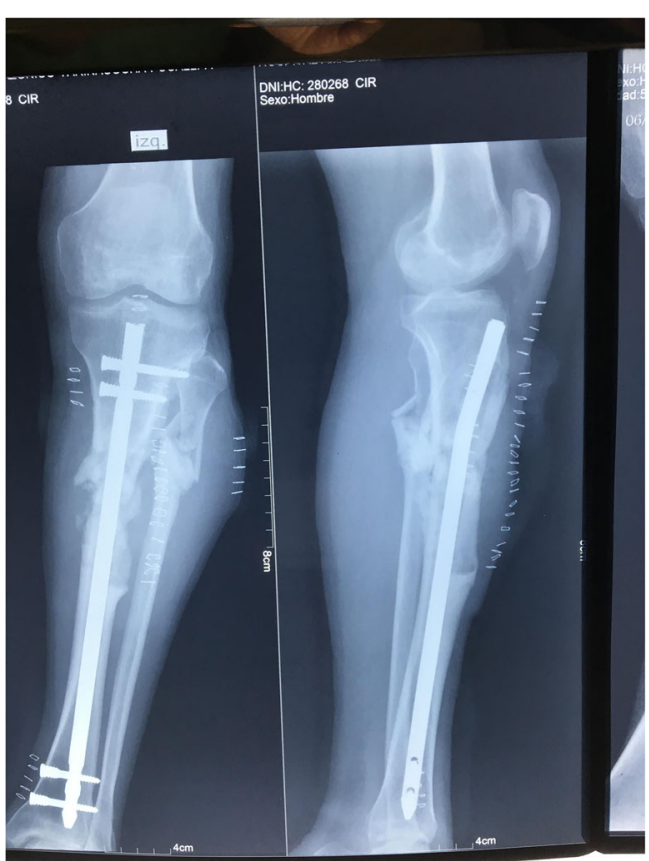

Fig. 8 Post operative radiograph with SIGN nail in place. Intraoperative photo is also shown in Fig. 5

volume and the availability of blood products in a given hospital. In hospitals without blood transfusion capabilities, keeping patients in traction until their hematocrit has returned to a safe level is a viable option and should be factored into the decision-making process regarding the timing of the procedure and the method of reduction and fixation.

All surgeons who participate in the SIGN program receive the necessary training to perform SIGN nailing safely.

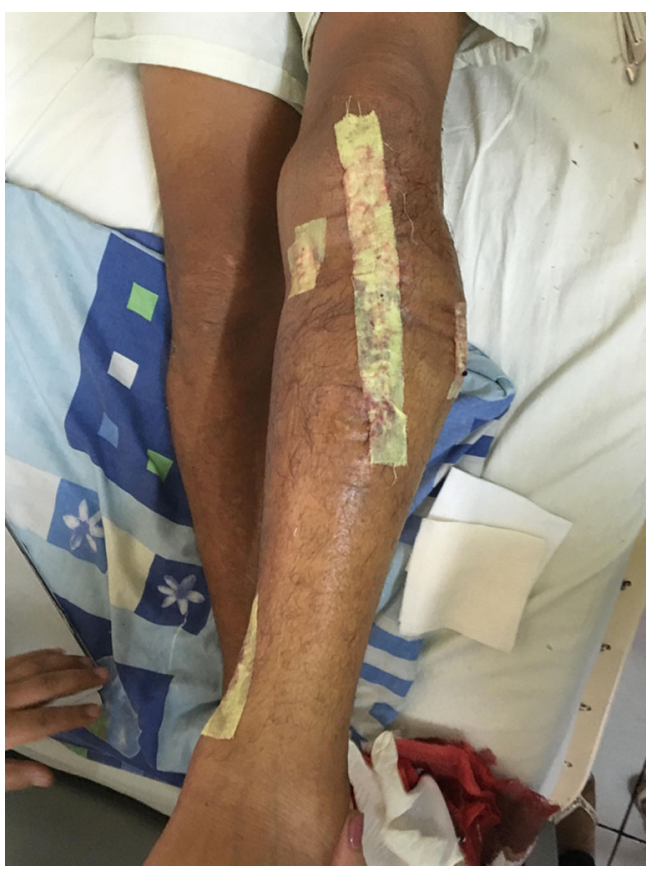

Fig. 9 Clinical photo on post operative day [1] 
Training occurs at the annual SIGN conference held in Richland, WA, as well as courses in Tanzania, Afghanistan, Vietnam, the Philippines, and other SIGN sites throughout the world. In the early years, all training was performed directly by Dr. Zirkle at the surgeon's home hospital. As the number of surgeons within the SIGN network grew, training has expanded to instruction by visiting SIGN-certified humanitarian surgeons or training by the regional SIGN expert. Currently, there are 52 countries with nearly 300 SIGN programs. Five thousand surgeons are trained in the SIGN technique and have performed almost 165,000 surgeries.

Another critical component of the SIGN program addresses both the recurring costs of the hardware and the need to track and analyze outcomes with this technique. SIGN nails and instrumentation are provided at no cost to surgeons in the developing world as long as they participate in the SIGN online surgical database (SOSD) to track and report their outcomes. In addition to the operating surgeons, there are direct cost savings to the patients as surgeons or hospitals are not allowed to charge patients for the SIGN implants.

\section{Outcomes with the SIGN Nail}

The SOSD is the largest database on orthopedic trauma in low and middle-income countries. Recent outcomes and costeffectiveness articles have been published to support the use of the SIGN nail [15, 21••, 22-24].

Young et al. reviewed 34,361 surgeries from the SIGN data base prior to 2010 to evaluate infection risk [21••]. Overall, follow-up evaluations were available on only $18 \%$ of cases, which reflects the challenge in LMICs for patients who lack the means or finances to travel long distances for a follow-up evaluation, particularly if they are asymptomatic. Among the cohort that did have follow-up data available, the infection rates in the tibia were $1.7 \%$ and in the femur $0.7 \%$. The authors performed a regression analysis to add validity to the study, creating a "worse case" estimation of infection rates at $7.3 \%$ for tibias and $3.5 \%$ for femurs. Even with this result, the study concluded that overall infection rates with the SIGN nail were similar to those in developed nations using standard intramedullary nailing systems.

In 2011, Sekimpi published his case series from Kampala, Uganda of 50 SIGN nailing surgeries for femoral shaft fractures in The Journal of Bone and Joint Surgery [22]. His union rate was $100 \%$ by 6 months with two patients requiring nail dynamization by removal of the interlocking bolts. Four patients healed with greater than $10^{\circ}$ of angular deformity and were classified as malunions. There was one superficial and one deep infection in his series.

Gosselin performed a cost and effectiveness study on the first cohort of 50 SIGN nails performed in a rural Cambodian hospital, comparing them to the prior 50 femur fractures treated in Perkin's traction [15]. The results showed a significant cost savings, improved outcomes, and a low infection rate. Hospital stays continued to decrease after the study as physicians, physical therapists, and patients became more confident in early mobilization.

\section{Conclusions}

Humanitarian fracture care is an exciting challenge for surgeons interested in helping the $2 / 3$ of the world's population that has little or no access to orthopedic treatment. The SIGN organization was founded on the belief that all people should have access to orthopedic care. SIGN provides surgeons in developing nations the education and training necessary to treat complex fractures as well as the implants for managing long bone fractures of the femur and tibia at no cost. The effectiveness and safety of the SIGN technique is evaluated by the SIGN database.

The SIGN nail technique requires surgeons to master new skills, especially the steps required to place the interlocking screws at the end of the nail. These challenges are best learned with another surgeon experienced in the technique. The SIGN system is very effective at treating acute fractures up to 6 weeks old. Fractures that have gone onto nonunions or malunions require a greater degree of expertise and practice when utilizing the SIGN nail.

Future directions of the SIGN organization are further development of implants to treat periarticular fractures of the hip and knee and education and training in deformity correction for malunion and nonunion problems and in spine and pelvic surgery.

\section{Compliance with Ethical Standards}

Conflict of Interest The author has been a Board Member for the SIGN organization since 2015 .

Human and Animal Rights and Informed Consent This article does not contain any studies with human or animal subjects performed by the author.

\section{References}

Papers of particular interest, published recently, have been highlighted as:

- Of importance

•. Of major importance

1. Mathers CD, Loncar D. Projections of global mortality and burden of disease from 2002 to 2030. PLoS Med. 2006;3, e442. doi:10. 1371/journal.pmed.0030442. 
2. Lopez AD, Mathers CD, Ezzati M, Jamison DT, Murray CJL (2006) Measuring the global burden of disease and risk factors, 1990-2001. In: Lopez AD, Mathers CD, Ezzati M, Jamison DT, Murray CJL, eds. Global burden of disease and risk factors. The International Bank for Reconstruction and Development/The World Bank, Washington, DC; Oxford University Press, New York, 1:1-14.

3. Gosselin RA, Spiegel DA, Coughlin R, Zirkle LG. Injuries: the neglected burden in developing countries. Bull World Health Organ. 2009;87(4):246-246a.

4. Gosselin, Richard A. Cost-effectiveness of orthopedic surgery in austere environments, Chapter 4, page 37 in Global Orthopedics, Springer 2014.

5. Richard C. Fisher, Richard A. Gosselin, Michelle Foltz. Nonsurgical principles of fracture and injury management Chapter 13, page 75 in Global orthopedics, Springer 2014.

6. Buxton RA. The use of Perkins' traction in the treatment of femoral shaft fractures. JBJS Br. 1981;63-B(3):362-6.

7. Gosselin RA, Lavaly DJ. Perkins traction for adult femoral shaft fractures: a report on 53 patients in Sierra Leone. Int Orthop. 2007;31(5):697-702.

8. Webb LX, Gristina AG, Fowler HL. Unstable femoral shaft fractures: a comparison of interlocking nailing versus traction and casting methods. J Orthop Trauma. 1988;2(1):10-2. doi:10.1097/ 00005131-198802000-00003.

9. Carr CR, Wingo CH. Fractures of the femoral diaphysis. A retrospective study of the results and costs of treatment by intramedullary nailing and by traction and a spica cast. J Bone Joint Surg Am. 1973;55(4):690-700.

10. Johnson KD, Johnston DW, Parker B. Comminuted femoral shaft fractures: treatment by roller traction, cerclage wires and an intramedullary nail, or an interlocking intramedullary nail. J Bone Joint Surg Am. 1984;66(8):1222-35.

11. Downing ND, Griffin DR, Davis TR. A comparison of the relative costs of cast treatment and intramedullary nailing for tibial diaphyseal fractures in the UK. Injury. 1997;28:373-5. doi:10.1016/ S0020-1383(97)00028-4.

12. Toivanen JA, Hirvonen M, Auvinen O, et al. Cast treatment and intramedullary locking nail for simple and spiral wedge tibial shaft fractures - a cost-benefit analysis. Ann Chir Gynaecol. 2000;89(2): $138-42$.

13. Gillquist J, Liliedahl SO, Rieger A. Fracture of the femur diaphysis. traction or nailing? Acta Chir Scand. 1971;137(6):515-20.

14. Fakhry SM, Rutledge R, Dahners LE, Kessler D. Incidence, management, and outcome of femoral shaft fracture: a statewide populationbased analysis of 2805 adult patients in a rural state. J Trauma. 1994;37(2):255-60. doi:10.1097/00005373-199408000-00018.

15. Gosselin RA, Heitto M, Zirkle L. Cost-effectiveness of replacing skeletal traction by interlocked intramedullary nailing for femoral shaft fractures in a provincial trauma hospital in Cambodia. Int Orthop (SICOT). 2009;33:1445-8.

16. Kamau DM, MBChB, MMed (Ortho Surg), Gakuu LN, MBChB, MMed (Surg), FCS (ECSA), Professor, Gakuya EM, MBChB, MMed (Surg), FCS (ECSA), Lecturer and Sang EK, MBChB, MMed Comparison of closed femur fracture: skeletal traction and intramedullary nailing cost-effectiveness. East African Orthopaedic Journal. EAOJ; Vol. 8: March 2014, pp. 4-9.

17. Phillips J, Zirkle LG, Gosselin RA. Achieving locked intramedullary fixation of long bone fractures: technology for the developing world. Int Orthop. 2012;36(10):2007-13. This article nicely summarizes the development of the SIGN Organization and its impact on the treatment of orthopaedic trauma worldwide.

18. Foltz M. A leg to stand on. iUniverse Press, 2009

19. Calafi LA, Antkowiak T, Curtiss S, Neu CP, Moehring DA. A biomechanical comparison of the Surgical Implant Generation Network (SIGN) tibial nail with the standard hollow nail. Injury. 2010;41(7):753-7. doi:10.1016/j.injury.2010.03.003.

20. Bishop JA, Dikos GD, Mickelson D, Barei DP. Open reduction and intramedullary nail fixation of closed tibial fractures. Orthopedics. 2012;35(11):e1631-4. doi:10.3928/0147744720121023-21.

21.• Young S, Lie SA, Hallan G, Zirkle LG, Engesaeter LB, Havelin LI. Low infection rates after 34,361 intramedullary nail operations in 55 low- and middle-income countries: validation of the Surgical Implant Generation Network (SIGN) online surgical database. Acta Orthop. 2011;82(6):737-43. doi:10.3109/17453674.2011. 636680. This article was the first rigorously performed outcome study on the SIGN database that confirmed the safety and efficacy of the SIGN nailing technique.

22. Sekimpi P, Okike K, Zirkle L, Jawa A. Femoral fracture fixation in developing countries: an evaluation of the Surgical Implant Generation Network (SIGN) intramedullary nail. J Bone Joint Surg Am. 2011;93(19):1811-8. doi:10.2106/JBJS.J.01322.

23. Ikem IC, Ogunlusi JD, Ine HR. Achieving interlocking nails without using an image intensifier. Int Orthop. 2007;31(4):487-90. doi: 10.1007/s00264-006-0219-3.

24. Shearer D, Cunningham B, Zirkle LG. Population characteristics and clinical outcomes from the SIGN online surgical database. Technol Orthop. 2009;24(4):273.

\section{Recommended Reading}

25.• Gosselin RA, Spiegel Michelle Foltz DA editors. Global Orthopedics. Caring for musculoskeletal conditions and injuries in austere settings. Springer, 2014. This recent textbook provides an extensive background on international orthopedics for those interested in working in a resource poor environment. It provides history and background, practical advice on both surgical and non surgical treatment of orthopedic conditions and useful chapters on understanding cultural differences that can mean the difference between success and failure when working abroad.

\section{Additional Information}

Surgeons interested in learning the SIGN technique or meeting international surgeons from the 50 countries participating in the SIGN network are encouraged to attend the SIGN conference that is held annually in Richland, WA, or to contact SIGN through the website at www. signfracturecare.org.

Dr. Zirkle's journey in humanitarian orthopedics is eloquently detailed in the book, A Leg to Stand On, by Michelle Foltz, MD. There is also abundant information on SIGN's mission to educate and bring equality of fracture care to the poor at www.signfracturecare.org. 